\title{
Joint Optimisation of Resampling Rate and Carrier-to-Signal Power Ratio in Direct-Detection Kramers-Kronig Receivers
}

\author{
Z. Li, M.S. Erkılınç, K. Shi, E. Sillekens, L. Galdino, B.C. Thomsen, P. Bayvel, and R.I. Killey
}

Optical Networks Group, Department of Electronic \& Electrical Engineering, UCL (University College London), London, WC1E 7JE, UK, zhe.li@ee.ucl.ac.uk

\begin{abstract}
We investigate the effect of varying the DSP resampling rate and the carrier-to-signal power ratio on the performance of direct-detection Kramers-Kronig receivers, through experiments on $4 \times 112 \mathrm{~Gb} / \mathrm{s}$ SSB Nyquist-SCM transmission over $240 \mathrm{~km}$.
\end{abstract}

\section{Introduction}

The ever-increasing traffic growth within metro, backhaul and inter-data centre networks brings the requirement for high-capacity, spectrallyefficient and low-cost optical transceivers. Since a massive number of transceivers are utilized within these applications, it would be expensive to utilise polarization-multiplexed coherent systems throughout. Single polarization single photodiode-based direct-detection (DD) transceivers are attractive for application scenarios of $\geq 100 \mathrm{~Gb} / \mathrm{s}$ per channel transmission over up to several hundred kilometres. For such systems, single-sideband (SSB) quadrature amplitude modulation (QAM) subcarrier modulation (SCM) can be utilized to achieve high spectral efficiency (SE). However, signal-signal beat interference (SSBI), a nonlinear effect due to squarelaw detection, severely degrades the performance of such systems.

The Kramers-Kronig (KK) receiver has been recently proposed to overcome this problem ${ }^{1}$. If minimum phase single-sideband (SSB) signalling is employed, the KK scheme allows the accurate reconstruction of the optical phase of the transmitted signal using only the detected signal's amplitude. It has been demonstrated for SSB DD systems through numerical simulations ${ }^{1}$ and experiments ${ }^{2,3}$, confirming its potential superiority in minimising the nonlinear penalty compared to previously proposed SSBI cancellation techniques ${ }^{4}$. In this paper, we investigate the key aspects of its practical implementation, namely the optimisation of the utilized optical carrier-to-signal power ratio (CSPR) at the transmitter and the resampling rate in the receiver DSP.

\section{The Kramers-Kronig Scheme}

Fig. 1 shows the receiver DSP, including the Kramers-Kronig scheme and electronic dispersion compensation (EDC). Based on the Kramers-Kronig relation, provided the transmitted signal fulfils the condition of being minimum phase (assuming the optical signal is singlesideband with the optical carrier having an amplitude larger than that of the signal in the sideband), the phase of the transmitted signal, $\varphi(n)$, is linked to its amplitude, $h(n)$. Therefore, the complex-valued electric field of the transmitted SSB signal can be extracted from the measured photocurrent following the directdetection. The KK scheme is written as:

$$
\begin{gathered}
h(n)=\operatorname{sqrt}\left(V_{D D}(n)\right) . \\
\varphi(n)=\mathcal{F}^{-1}\{i \cdot \operatorname{sign}(\omega) \mathcal{F}\{\ln [|h(n)|]\}\} \\
V_{K K}(n)=h(n) \cdot \exp \{i \varphi(n)\} .
\end{gathered}
$$

where $V_{D D}(n)$ is the detected real-valued doublesideband (DSB) signal, $n$ is the discrete time index, $\operatorname{sign}(\omega)$ is the sign function, which is equal to 1 for $\omega>0$, to 0 for $\omega=0$, and to -1 for $\omega<0$, and $\mathcal{F}\{\bullet\}$ and $\mathcal{F}^{-1}\{\bullet\}$ are the Fourier and inverse Fourier transform operators. In the KK scheme's algorithm, due to the high bandwidth of the signals resulting from the square-root and logarithm operations, it is necessary to utilize a relatively high oversampling rate.

From the above discussion, it can be seen that two key conditions need to be fulfilled to achieve optimum performance of the KK scheme: firstly, the transmitted SSB signal should have a sufficiently high power optical carrier (i.e. a sufficiently high CSPR value) to ensure minimum phase signalling, and, secondly, digital upsampling to deal with the bandwidth

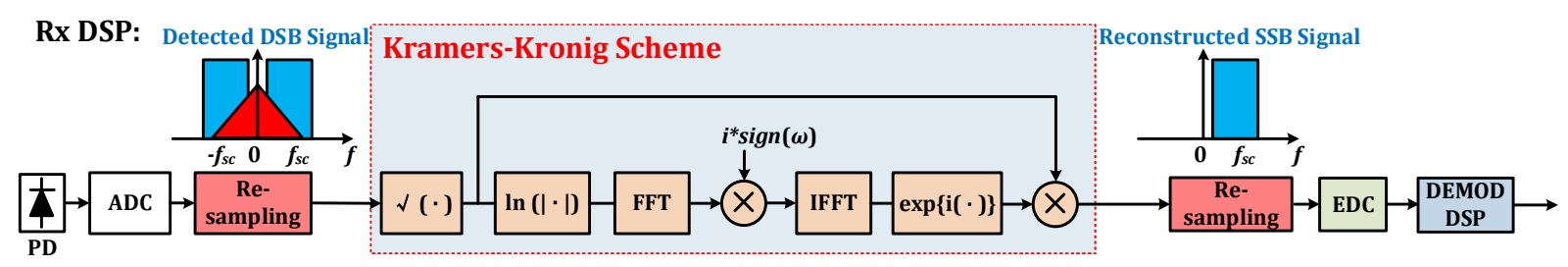

Fig. 1: Receiver DSP with KK scheme. PD: Photodiode. ADC: Analog-to-digital converter. EDC: Electronic dispersion compensation. DEMOD DSP: Demodulation DSP for SSB SCM signal. 


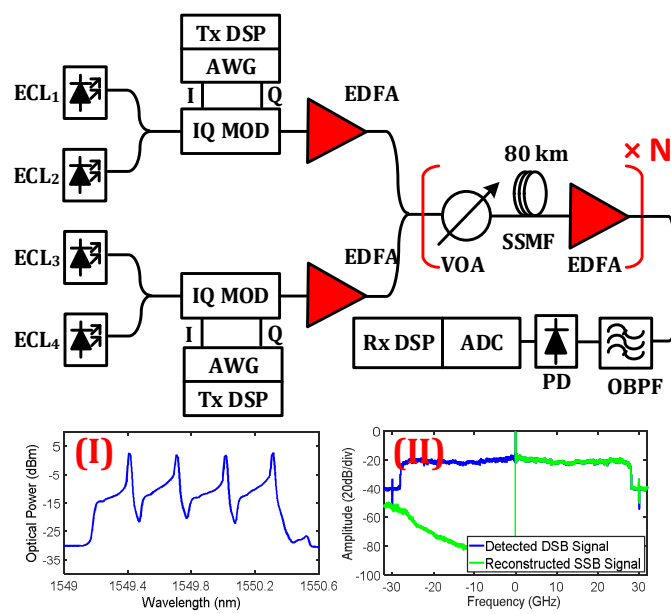

Fig. 2: Optical transmission experimental testbed Insets: (I) experimental transmitted WDM spectrum. (II) detected digital DSB signal spectrum (blue line) and reconstructed digital SSB signal spectrum (green line) obtained using the KK scheme. ECL: External cavity laser. AWG: Arbitrary waveform generator. EDFA: Erbium-doped fiber amplifier. VOA: Variable optical attenuator. SSMF: Standard single-mode fiber. OBPF: Optical band-pass filter. PD: Photodiode.

broadening effect must be carried out. In our experiments, which were assessed with a spectrally-efficient $(3.18 \mathrm{~b} / \mathrm{s} / \mathrm{Hz}) \quad 4 \times 112 \mathrm{~Gb} / \mathrm{s}$ WDM DD SSB 16-QAM Nyquist-SCM system, the CSPR and DSP resampling rate were swept, to identify the values for optimum performance.

\section{Experimental Setup}

The experimental setup is shown in Fig. 2. At the transmitter, two IQ-modulators seeded by four external cavity lasers (ECLs) were utilized to generate the odd and even channels. The modulators were driven by two arbitrary waveform generators (AWGs) operating at $92 \mathrm{GSa} / \mathrm{s}$ with $33 \mathrm{GHz} 3-\mathrm{dB}$ bandwidth. In the Tx DSP, a $28 \mathrm{GBd}(112 \mathrm{~Gb} / \mathrm{s})$ SSB 16-QAM Nyquist-SCM signal was generated with a subcarrier frequency of $14.28 \mathrm{GHz}(0.51 \times$ symbol rate). Following this, a $35 \mathrm{GHz}$-spaced $4 \times 112 \mathrm{~Gb} / \mathrm{s}$ WDM signal (Fig. 2 inset (I)) was generated by multiplexing the modulated odd and even channels, giving a gross optical SE of $3.2(\mathrm{~b} / \mathrm{s}) / \mathrm{Hz}$. The CSPR value was optimized at each value of optical signal-to-noise ratio (OSNR) to achieve the optimum performance. Transmission experiments were carried out using a straight-line multiple span fibre link with $80 \mathrm{~km}$ span length with SSMF and erbium-doped fibre amplifiers (EDFA) with a $5 \mathrm{~dB}$ noise figure. At the receiver, a $31 \mathrm{GHz}$ 3-dB bandwidth optical band-pass filter (OBPF) was applied to demultiplex the channel of interest. A single PIN photodiode followed by a single ADC operating at $80 \mathrm{GSa} / \mathrm{s}$ were utilized to perform signal detection and digitization. The detected real-valued DSB signal's spectrum is shown in Fig. 2 inset (II). Following this, in the Rx DSP (Fig. 1), the detected signal was resampled from the ADC rate $(80 \mathrm{GSa} / \mathrm{s})$ to a value of between 2 and 9 $\mathrm{Sa} / \mathrm{symbol}$ (56 GSa/s to $252 \mathrm{GSa} / \mathrm{s}$ ) at which rate the KK algorithm was performed, following which it was down-sampled to $2 \mathrm{Sa} /$ symbol before the EDC and demodulation DSP were performed. The reconstructed SSB signal spectrum after the KK scheme is plotted in Fig. 2 inset (II).

\section{Experimental Results}

Both the optical back-to-back and WDM transmission performance were evaluated by varying the CSPR value at the transmitter and the resampling rate for the $\mathrm{KK}$ scheme at the receiver. The required OSNR (assuming BER = $3.8 \times 10^{-3}$ ) versus CSPR in back-to-back operation is plotted in Fig. 3(a). At a fixed resampling rate, a trade-off between the nonlinear and linear penalties can be clearly observed. Signals operating at lower CSPR values suffer from large nonlinear penalty, since the minimum phase condition is not met, while higher CSPR values lead to higher linear penalty (increased carrier-ASE beating). On the other hand, the required OSNR values increase at lower resampling rates, due to their failure to meet the Nyquist criterion for spectrally broadened signals generated within the $\mathrm{KK}$ algorithm. The

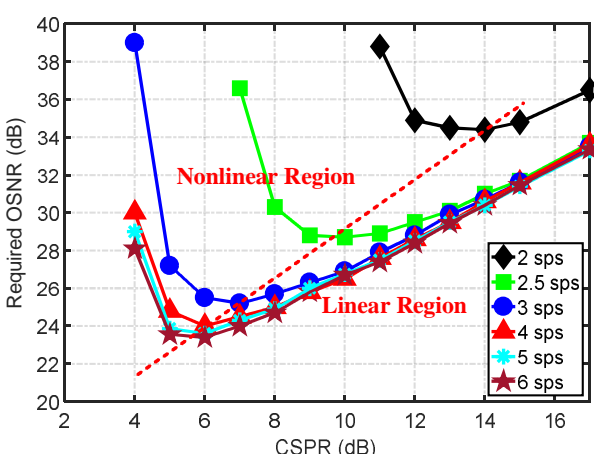

(a)

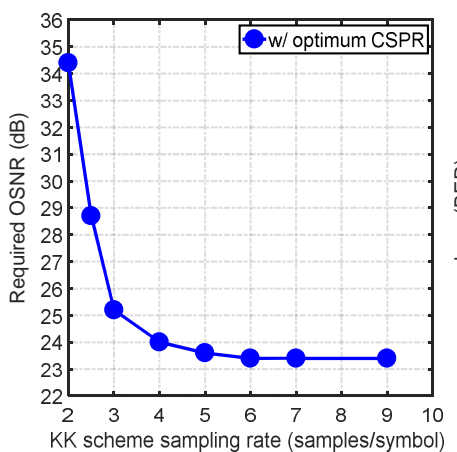

(b)

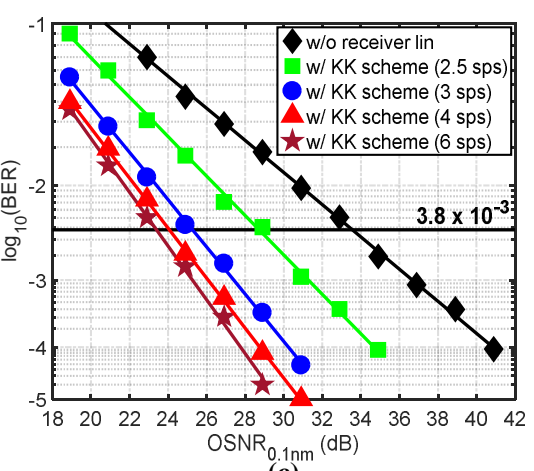

(c)

Fig. 3: System back-to-back performance with the KK scheme: (a) Required OSNR (assuming BER $=3.8 \times 10^{-3}$ ) versus CSPR. (b) Required OSNR at the optimum CSPR value versus KK scheme resampling rate. (c) BER versus OSNR. 


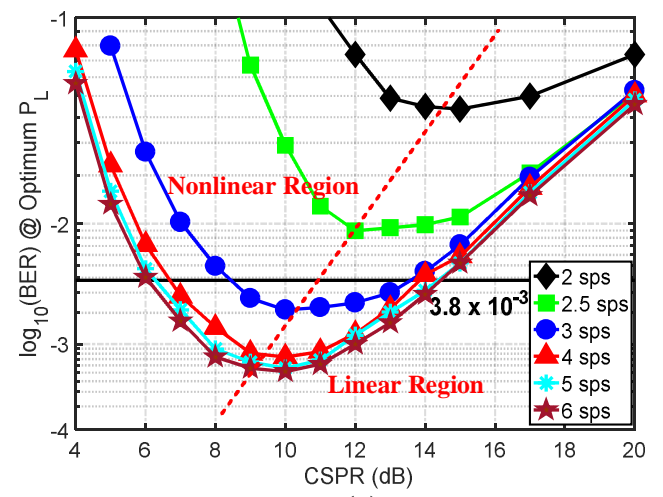

(a)

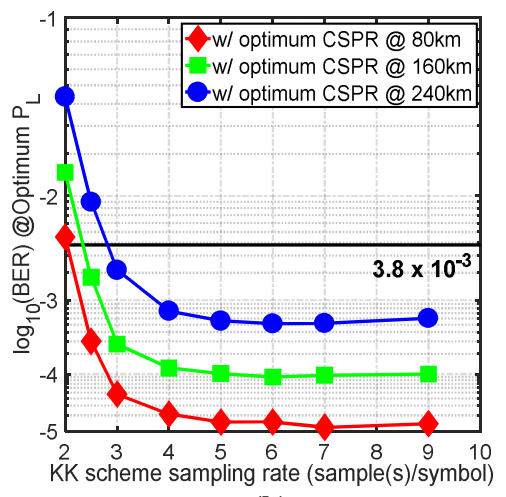

(b)

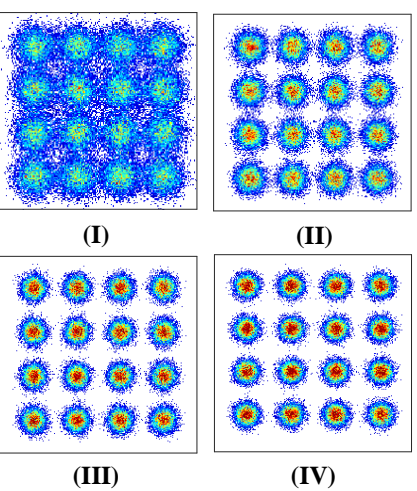

(III)

(IV)

Fig. 4: System transmission performance with the KK scheme: (a) BER at the optimum optical launch power versus CSPR at 240 $\mathrm{km}$. (b) BER at the optimum launch power versus resampling rate at 80, 160 and $240 \mathrm{~km}$. Insets: Received constellations at 240 $\mathrm{km}$ with (I) $2.5 \mathrm{Sa} / \mathrm{symbol}(\mathrm{EVM}=22.4 \%)$, (II) $3 \mathrm{Sa} / \mathrm{symbol}(\mathrm{EVM}=16.7 \%)$, (III) $4 \mathrm{Sa} / \mathrm{symbol}(\mathrm{EVM}=14.2 \%)$ and (IV) $6 \mathrm{Sa} / \mathrm{symbol}$ $(E V M=13.6 \%)$

optimum CSPR shifts to higher values at lower sampling rates. The required OSNRs at the optimum CSPR values over a range of resampling rates are plotted in Fig. 3(b). When the resampling rate is lower than $4 \mathrm{Sa} /$ symbol (112 GSa/s), a significant increase in the required OSNR (from $24 \mathrm{~dB}$ at $4 \mathrm{Sa} / \mathrm{symbol}$ to $34.4 \mathrm{~dB}$ at $2 \mathrm{Sa} /$ symbol) is observed; the performance converges $(23.4 \mathrm{~dB}$ required OSNR) when utilizing $6 \mathrm{Sa} / \mathrm{symbol}(168 \mathrm{GSa} / \mathrm{s})$ or more. The BER versus OSNR is plotted in Fig. 3 (c). The required OSNR at the $3.8 \times 10^{-3}$ BER threshold was found to be $33.5 \mathrm{~dB}$ without using receiver linearization scheme, reducing by 10.1 $\mathrm{dB}$ to $23.4 \mathrm{~dB}$ with the $\mathrm{KK}$ scheme operating at $6 \mathrm{Sa} /$ symbol.

Next, the WDM transmission performance was assessed. Fig. 4(a) presents the BER at the optimum launch power versus CSPR following transmission over $240 \mathrm{~km}$. A trade-off between the nonlinear and linear effects can still be observed. With the lower resampling rates, the KK scheme's performance was degraded and the optimum CSPR values was increased. Fig. 4(b) shows the BER (at the optimum launch power and CSPR value) versus the utilized resampling rate at transmission distances of 80 , 160 and $240 \mathrm{~km}$. The BERs significantly decreased when resampling rates were increased from $2 \mathrm{Sa} /$ symbol to $4 \mathrm{Sa} / \mathrm{symbol}$, and converge for rates of $6 \mathrm{Sa} /$ symbols and above, with the minimum BERs found to be $1.5 \times 10^{-5}$, $9.9 \times 10^{-5}$ and $5.5 \times 10^{-4}$ at $80 \mathrm{~km}, 160 \mathrm{~km}$ and 240 $\mathrm{km}$ respectively. Furthermore, after transmission over $240 \mathrm{~km}$, the BER values for all the four WDM channels after implementing the KK scheme (6 Sa/symbol) were measured to be between $4.7 \times 10^{-4}$ and $5.7 \times 10^{-4}$, resulting in a net optical SE of $3.18 \mathrm{~b} / \mathrm{s} / \mathrm{Hz}$ based on the theoretical hard-decision decoding bound. As high resampling rate leads to increased DSP com- plexity, the sampling rate utilized in practical implementation of KK scheme may be lower than the theoretical optimum of $6 \mathrm{Sa} /$ symbol.

\section{Conclusions}

We presented, for the first time, an experimental study on the joint impact of the CSPR and resampling ratio on the performance of the $D D$ SSB Nyquist-SCM system employing the recently proposed Kramers-Kronig DSP scheme. The experimental analysis was carried out on a spectrally-efficient $(3.18 \mathrm{~b} / \mathrm{s} / \mathrm{Hz}) \quad 4 \times 112 \mathrm{~Gb} / \mathrm{s}$ WDM 16-QAM system. Good performance was achieved at $4 \mathrm{Sa} / \mathrm{symbol}$, with optimum performance at $\geq 6 \mathrm{Sa} / \mathrm{symbol}$. A low pre-FEC BER of $5.5 \times 10^{-4}$ in transmission over $240 \mathrm{~km}$ of uncompensated standard SMF was shown to be achievable. This analysis has quantified the trade-off between the linearization performance and DSP complexity when utilizing the KK scheme.

\section{Acknowledgements}

This work is supported by the UK EPSRC UNLOC project, and Semtech Corporation.

\section{References}

[1] A. Mecozzi, et al., "Kramers-Kronig coherent receiver," Optica, Vol. 3, no. 11, p. 1220-1227 (2016).

[2] Z. Li, et al., "SSBI mitigation and Kramer-Kronig scheme in single-sideband direct-detection transmission with receiver-based electronic dispersion compensation," J. Lightw. Technol., Vol. 35, no. 10, p. 1887-1893 (2017).

[3] X. Chen, et al., "218-Gb/s single-wavelength, singlepolarization, single-photodiode transmission over 125$\mathrm{km}$ of standard single-mode fiber using Kramers-Kronig detection", Proc. OFC, Th5B.6 (2017).

[4] Z. Li, et al., "Comparison of digital signal-signal beat interference compensation techniques in direct-detection subcarrier modulation systems," Opt. Express, Vol. 24, no. 25 , p. 29176-29189 (2016). 